\title{
Manipulating the Rashba-type spin splitting and spin texture of Pb quantum well states
}

\author{
Bartosz Slomski, ${ }^{1,2}$ Gabriel Landolt, ${ }^{1,2}$ Fabian Meier,,${ }^{1,2}$ Luc Patthey, ${ }^{2}$ Gustav Bihlmayer, ${ }^{3}$ Jürg Osterwalder, ${ }^{1}$ and J. Hugo Dil ${ }^{1,2}$ \\ ${ }^{1}$ Physik-Institut, Universität Zürich, Winterthurerstrasse 190, CH-8057 Zürich, Switzerland \\ ${ }^{2}$ Swiss Light Source, Paul Scherrer Institut, CH-5232 Villigen, Switzerland \\ ${ }^{3}$ Peter Grünberg Institut and Institute for Advanced Simulation, Forschungszentrum Jülich and JARA, DE-52425 Jülich, Germany
}

(Received 8 June 2011; revised manuscript received 11 October 2011; published 16 November 2011)

\begin{abstract}
Using spin- and angle-resolved photoemission spectroscopy we show that the spin splitting and the spin texture of quantum well states in thin $\mathrm{Pb}$ films can be manipulated through changes in the interface to the substrate. Compared with films grown on the $\mathrm{Pb}$ reconstructed $\mathrm{Si}(111)$ substrate the $\mathrm{Bi}$ interface reduces the spin splitting of the $\mathrm{Pb}$ states by a factor of 2 and the spin polarization vector is rotated by $32^{\circ}$ out of the sample plane. The spin splitting on an Ag reconstructed substrate is below the experimental resolution. Based on a model supported by ab initio calculations we interpret these changes as due to a modification of the charge density distribution in the quantum well states.
\end{abstract}

DOI: 10.1103/PhysRevB.84.193406

PACS number(s): 73.21.Fg, 71.70.Ej, 79.60.Dp

Spin-orbit interaction (SOI) in low-dimensional nonmagnetic materials, with the resulting spin-momentum locking via the Rashba effect, ${ }^{1}$ is one of the key ingredients for designing a field effect transistor that utilizes the electron's spin as a control parameter, rather than its charge. ${ }^{2}$ Along the long pathway toward functional devices the question of whether we can manipulate the spin texture is of particular interest: ultimately one would like to be able to control the magnitude of the spin splitting and the spin direction of a Rashba-type system through an easily accessible external parameter.

To date, several fields in condensed matter physics have tried to address this question. In semiconductor heterostructures, for instance InGaAs/InAlAs, a gate-controlled spin splitting was shown. ${ }^{3}$ However, in those systems the splitting is extremely small. Another class of materials showing Rashbatype spin splitting are given by noble metal surfaces and surface alloys. ${ }^{4-6}$ Many insights into the origin of the strength of spin-orbit interaction have been achieved by studying these systems. ${ }^{7}$ However, due to the metallic substrates they are not suitable for applying an electric field. On the technologically more relevant semiconductor substrates a significant spin splitting has been measured after deposition of heavy metals in monolayer quantity to form surface reconstructions, but these systems are very sensitive to contaminations and show no clear prospect of tunability. ${ }^{8,9}$ Quantum well states (QWS) in thin metal films ${ }^{10}$ confined between an absolute band gap of a semiconducting substrate and the repulsive image potential in front of the surface combine many of the missing aspects.

It was shown that the Rashba effect in Pb QWS occurs throughout the whole layer as a result of competing effects between the metal-substrate and metal-vacuum interfaces. ${ }^{11}$ This is indicated by the reversed spin helicity compared to the surface state of $\mathrm{Au}(111)$ and the lack of thickness dependence of the strength of Rashba-type spin splitting. Like in the case of metal surface states, the strength of the Rashba splitting in this system is mainly determined by the asymmetric charge distribution around the nuclei. ${ }^{12}$ However, the charge density of QWS is distributed over the entire film and has tails penetrating the confining barriers, both at the surface and at the film-substrate interface. Consequently the Rashba effect in QWS, as measured by spin and angle-resolved photoemission spectroscopy (SARPES), has contributions from local spinorbit splittings arising at each atomic layer of the ultrathin $\mathrm{Pb}$ film.

In this work we study the influence of the metal-substrate interface of ultrathin $\mathrm{Pb}$ films on the spin splitting of $\mathrm{Pb}$ QWS, comparing the results from a $\mathrm{Bi}$, an $\mathrm{Ag}$, and a $\mathrm{Pb}$ reconstructed $\mathrm{Si}(111)$ substrate. For these systems the metalvacuum boundary conditions are the same.

Figure 1(a) shows an in-plane integrated charge density distribution $\left[|\psi(z)|^{2}\right]$ of a Pb QWS in a 10 monolayer (ML) thick $\mathrm{Pb}$ slab on $\mathrm{Si}(111)$ for $k_{\|}=0.1 \overline{\Gamma K}$.

The charge density distribution shows a typical quantum beat structure due to the fast oscillating Bloch factor accounting for the slab periodicity and the standing wave solution with $n=5$ (number of antinodes). In the lower panel of Fig. 1(a) we show the layer-resolved spin-orbit splittings, which were obtained by artificially turning off the SOI in the other layers. At each layer $\left(L_{i}\right)$ a local atomic contribution $\left(\Delta E_{i}\right)$ to the Rashba effect arises in the vicinity of the nuclei of the form: ${ }^{13} \Delta E_{i} \propto k_{\|} \int_{\Delta z} d z \frac{\partial V}{\partial z}|\psi(z)|^{2}$, with $V$ being the spherical Coulomb potentials of the indexed nuclei and $\psi$ is the wave function of the QWS. It has been shown that more than $90 \%$ of this contribution arise within $\Delta z \approx 0.5$ atomic units (a.u.) of the nuclei, where the antisymmetric Coulomb gradient $\partial_{z} V$ is most significant. ${ }^{12} \Delta E_{i}$ differs in magnitude and sign from layer to layer and strongly depends on the local charge density asymmetry. As shown in Fig. 1(b), at the layer $L_{3}\left(L_{7}\right)$ the QWS charge density intersects with the $\mathrm{Pb}$ core position, indicated by the dashed vertical line, at the falling (rising) slope, which yields a negative (positive) splitting of $\Delta E \approx 60 \mathrm{meV}$. At $L_{1}$ the local spin-orbit splitting is almost zero because $|\psi(z)|^{2}$ is here nearly symmetric. The sum of all local splittings is small because of the oscillatory behavior throughout the whole layer.

Figure 2(a) shows the spin-integrated band dispersion of a QWS in a $10 \mathrm{ML}(\approx 2.95 \mathrm{~nm})$ thick Pb film deposited on $\operatorname{Si}(111)-(\sqrt{3} \times \sqrt{3})-\operatorname{Bi}(\beta)$ [henceforth $\mathrm{Pb} / \mathrm{Bi} / \mathrm{Si}(111)]$. The $\beta$ phase described by the Bi trimer $\left(\mathrm{T}_{4}\right)$ model schematically drawn in Fig. 1(c), with a nominal coverage of $1 \mathrm{ML}$, was 
(a)

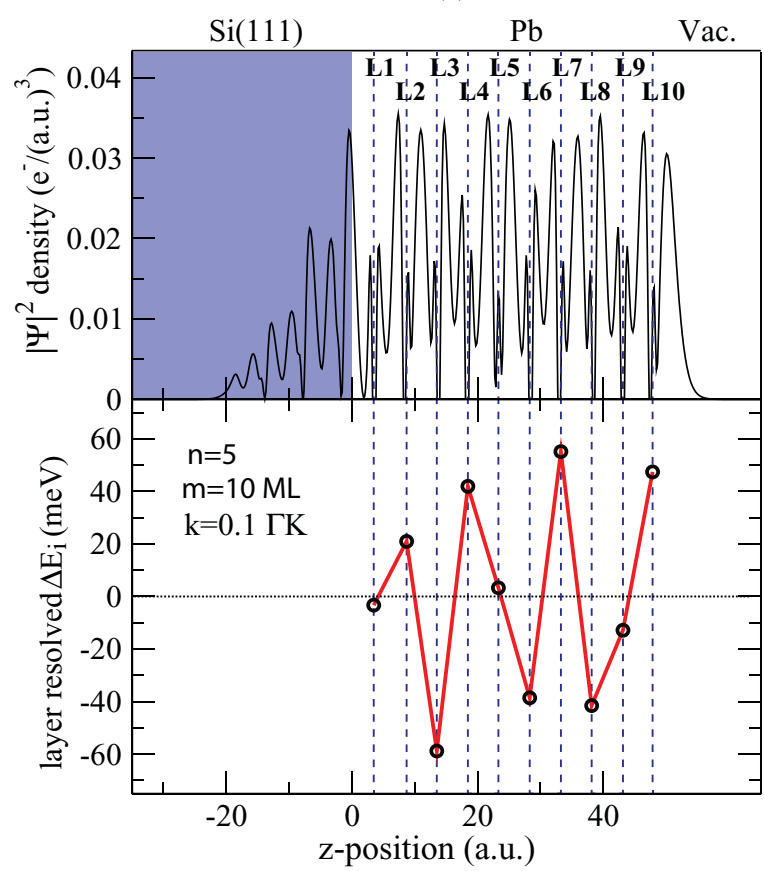

(b) $\Delta \mathrm{E}_{3}=-60 \mathrm{meV} \Delta \mathrm{E}_{7}=57 \mathrm{meV} \Delta \mathrm{E}_{1}=-4 \mathrm{meV}$

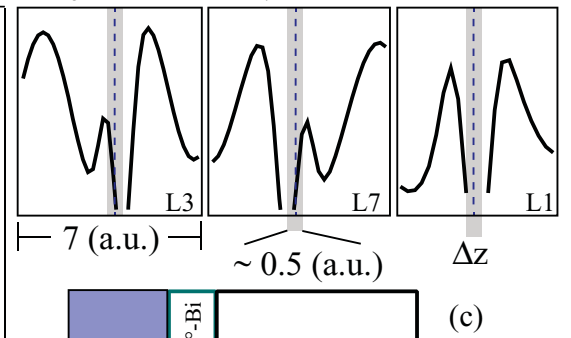

(c)

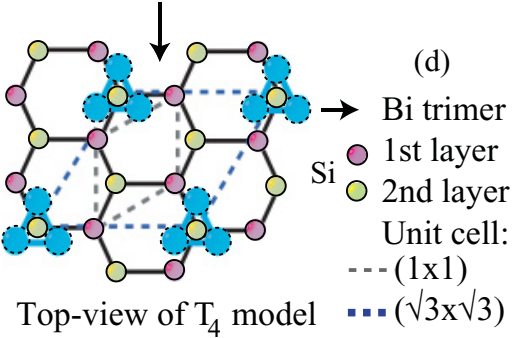

FIG. 1. (Color online) (a) Calculated in-plane averaged charge density of a QWS confined in an asymmetric 10 ML slab and (lower panel) layer resolved spin-orbit splittings. (b) Local charge density distribution at three specific nuclear positions of the slab. The shaded area indicates the region around the $\mathrm{Pb}$ core, which contribute to the local spin-orbit splitting. (c) Schematic drawing of the investigated ultrathin $\mathrm{Pb}$ film. (d) Top view of the $\mathrm{T}_{4}$ model of $\operatorname{Si}(111)-(\sqrt{3} \times \sqrt{3})-\operatorname{Bi}(\beta)$.

obtained by post-annealing a film of $3 \mathrm{ML}$ of $\mathrm{Bi}$ at $\approx 300^{\circ} \mathrm{C}$ for 1 min. ${ }^{14}$ The $(\sqrt{3} \times \sqrt{3})$-Ag interface was prepared in a similar way, except that the annealing temperature was at $\approx 500^{\circ} \mathrm{C}$. On both interfaces high quality $\mathrm{Pb}$ films were prepared by evaporation of $\mathrm{Pb}$ from a homemade Knudsen cell at low temperatures $(T<60 \mathrm{~K}){ }^{15}$ All measurements were performed with the COPHEE spectrometer at the Swiss Light Source of the Paul-Scherrer-Institute. ${ }^{16}$ The experimental measurement geometry of our study is sketched in Fig. 2(b). The energy and angular resolution of our spin-resolved ARPES measurements were $100 \mathrm{meV}$ and $\pm 0.75^{\circ}$. In all measurements a photon energy of $24 \mathrm{eV}$ was used because of the optimal photoemission cross section. ${ }^{17}$ More details about the growth and spin-integrated band structure of the Bi reconstructed system can be found elsewhere. ${ }^{18}$ The most important points are that the system features layer-by-layer growth and that the effective mass of the states around normal emission $\left(k_{\|}=0\right)$ is reduced by a factor of 3 to $m^{\star}=3.2 m_{e}$ compared to $\mathrm{Pb} / \mathrm{Pb} / \mathrm{Si}(111) .{ }^{19,20}$ This reduction is induced by the $\mathrm{Bi}$ interface and arises from a combination of the increased orbital overlap between the $6 p_{z}$ derived orbitals associated with the slightly smaller in-plane lattice constant, and hybridization of the downward dispersing $6 p_{x, y}$ bands with those of $6 p_{z}$ symmetry. ${ }^{18}$

Figure 2(c) shows the measured spin polarizations of $\mathrm{Pb} / \mathrm{Bi} / \mathrm{Si}$ in $y, x$, and $z$ directions $\left(P_{y}, P_{x}, P_{z}\right)$ of the sample coordinate system of an energy distribution curve (EDC) at the fixed reciprocal point $\mathbf{k}_{\|}=\left(k_{x}, k_{y}\right)=(0.24,0) \AA^{-1}$ along $\overline{\Gamma K}$ of the $(1 \times 1)$ surface Brillouin zone $(\mathrm{SBZ})$. From the Rashba model one expects up-down polarizations in the $y$ direction being tangential to the constant energy contour, as sketched in Fig. 2(f). The small $P_{y}$ amplitude of only $8 \%$ results from the large overlap between peaks of opposite spin direction and the influence of the background. The data were analyzed by a two-step fitting routine, which has proven to be a very powerful tool to extract three-dimensional polarization vectors. ${ }^{21}$ In short, the first step of this routine aims to fit the total spin-integrated intensity, as shown in Fig. 2(d). We define two Voigt intensity peaks (labeled as $R 1$ and $R 2$ ) and a linear background. The second step consists of the assignment of polarization vectors to $R 1$ and $R 2$, which are defined by their polar angles $\theta_{R i}, \phi_{R i}$ and length $c_{R i}=1$, with $i=1,2$. Peak widths, splittings, and spin polarizations are then varied until a self-consistent best fit to the intensity and polarization data is reached. The result of the two-step fit is shown in Fig. 2(d) for the intensity and in Fig. 2(c) for the polarization fit. In Fig. 2(e) we show spin-resolved intensities calculated as $I_{ \pm}^{y}=$ $\frac{I_{\text {tot }}}{2}\left(1 \pm P_{y}\right)$, where an energy splitting of $16 \mathrm{meV}$ between the spin up and down state can be seen and an individual peak width of $240 \mathrm{meV}$. Furthermore, the polarization vector is found to be rotated by $(32 \pm 2)^{\circ}$ out of the sample plane, in sharp contrast to the purely in-plane polarizations found for $\mathrm{Pb} / \mathrm{Pb} / \mathrm{Si}(111){ }^{11}$

In the following we will determine the change of the Rashba constant $\alpha_{\mathrm{RB}}=\hbar^{2} k_{0} / m^{\star}$ and the momentum splitting $k_{0}$ induced by the $\mathrm{Bi}$ interface. To address this issue, we have measured $P_{y}$ along $\overline{\Gamma M}$, that is, for $k_{y}=0 \AA^{-1}$ and different values of $k_{x}$, because for these momenta the spin quantization axis lies along the $y$ direction. The band dispersion of the investigated QWS (8 ML) and the corresponding scans are shown in Fig. 3(a).

As expected for a Rashba system we observe a $k$-dependent energy splitting. From the spin-polarized data in Fig. 3(b) the Rashba constant can be deduced by plotting the energy splitting, determined via the two-step fitting routine, versus 
(a)

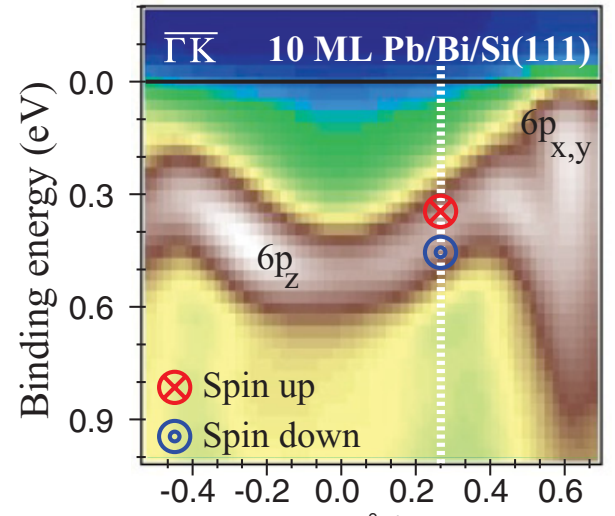

(c)

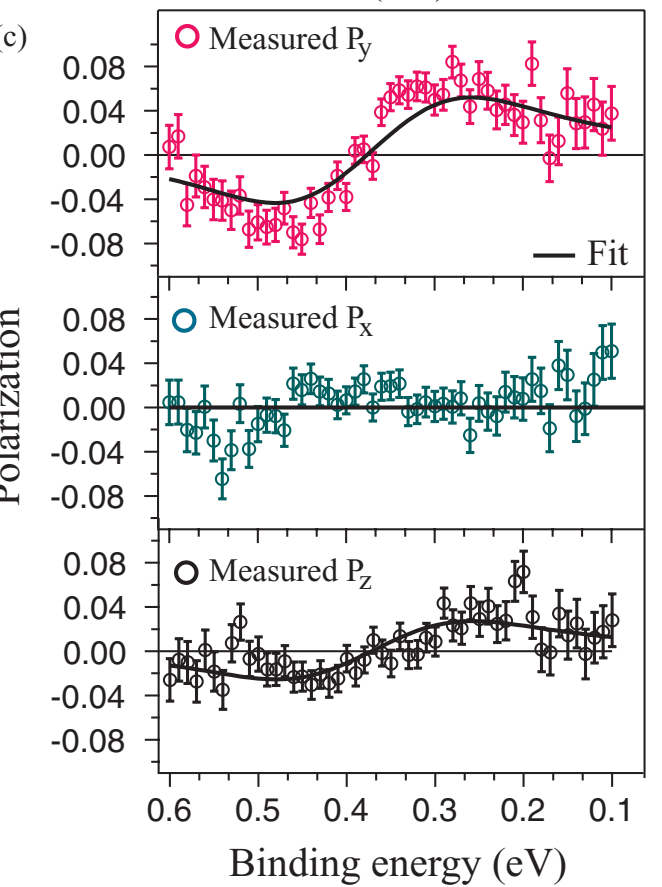

(f)

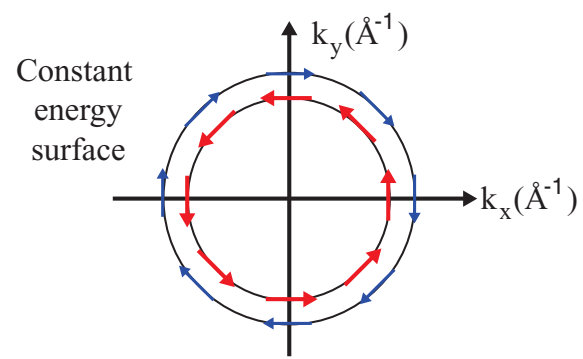

(b)
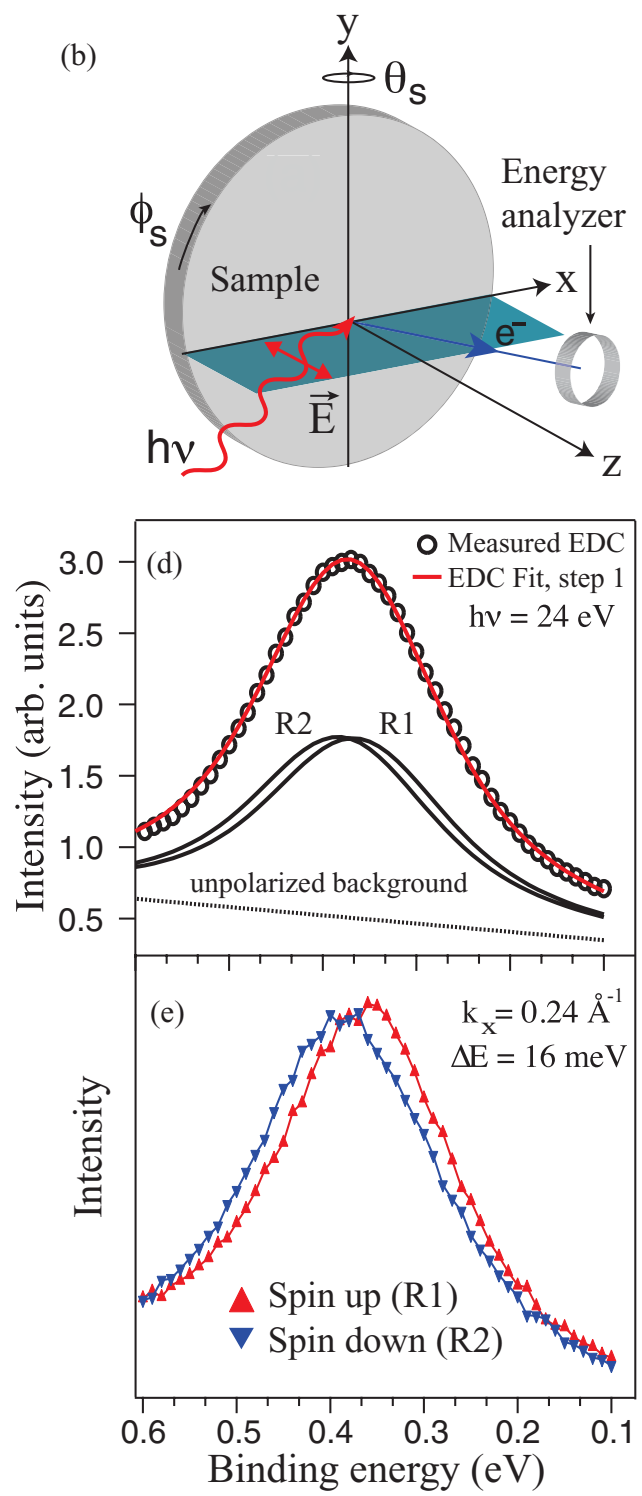

(g)

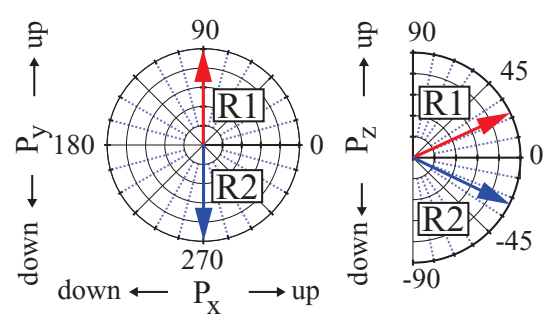

FIG. 2. (Color online) (a) Band dispersion of the investigated QWS in a $10 \mathrm{ML}$ thick $\mathrm{Pb}$ film grown on the Bi reconstructed substrate. (b) Experimental photoemission geometry of COPHEE. (c) Measured and fitted polarizations along $y, x$, and $z$ directions. (d) Measured spin-integrated intensity and the resulting fit. (e) Spin-resolved energy distribution curve. (f) Schematic constant energy surface of Pb/Bi/Si(111). (g) In-plane and out-of-plane polarization components at $k_{x}=0.24 \AA^{-1}$ and $k_{y}=0 \AA^{-1}$ obtained from the two-step fit.

in-plane momentum and taking the half of the slope of the linear fit, as shown in Fig. 3(c). We obtain a Rashba constant of $\alpha_{\mathrm{RB}, \mathrm{Bi}}=(0.032 \pm 0.005) \mathrm{eV} \AA$, which is here reduced by $60 \%$ compared to $\mathrm{Pb} / \mathrm{Pb} / \mathrm{Si}(111)$, where we find $\alpha_{\mathrm{RB}, \mathrm{Pb}}=(0.076 \pm 0.005) \mathrm{eV} \AA .^{22}$ This decrease implies that the $\mathrm{Bi}$ interface induces more symmetric local charge distributions around the $\mathrm{Pb}$ nuclei in the overlayer, or equivalently an overall more symmetric wave function envelope.

A smaller value of $\alpha_{\mathrm{RB}}$ can be interpreted as a smaller penetration of the wave function at the metal-substrate interface, because the metal-vacuum interface is the same for both systems. This conjecture is fully consistent with our interpretation of the decreased $m^{\star}$ in the sense that the $\mathrm{Bi}$ 

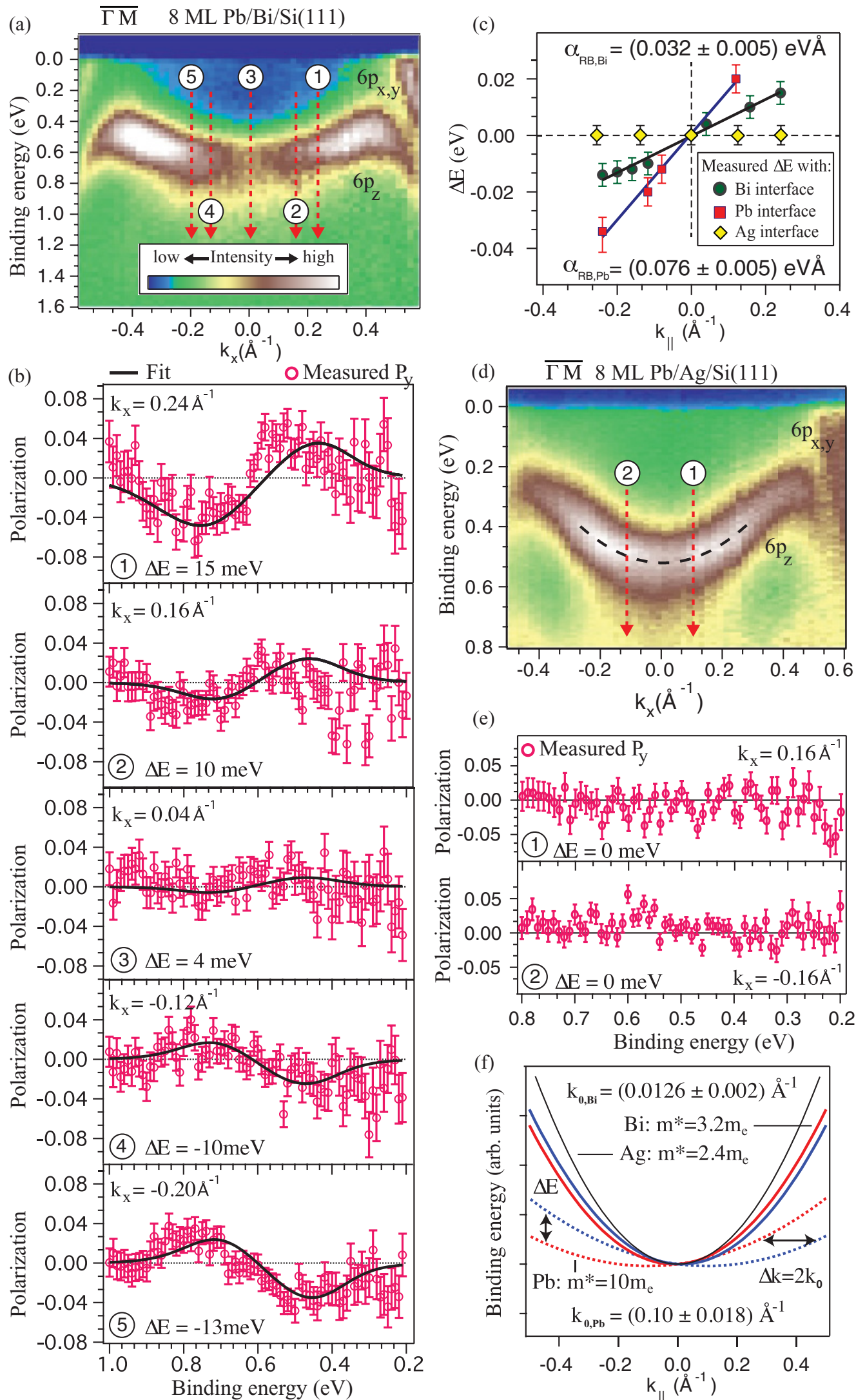

FIG. 3. (Color online) (a) Band dispersion of the investigated $8 \mathrm{ML} \mathrm{Pb}$ film on the Bi interface. (b) Measured and fitted $P_{y}$ spectra at $k_{y} \approx 0 \AA^{-1}$ and for several values of $k_{x}$. (c) Measured $k$-dependent energy splittings and linear fits (lines) to obtain $\alpha_{\mathrm{RB}}$ for the $\mathrm{Pb} / \mathrm{Pb} / \mathrm{Si}(111)$ and $\mathrm{Pb} / \mathrm{Bi} / \mathrm{Si}(111)$, respectively. (d) Band dispersion of $8 \mathrm{ML} \mathrm{Pb}$ film on the Ag interface. (e) Measured $P_{y}$ spectra. (f) Calculated spin-split parabola for the three studied interfaces. 


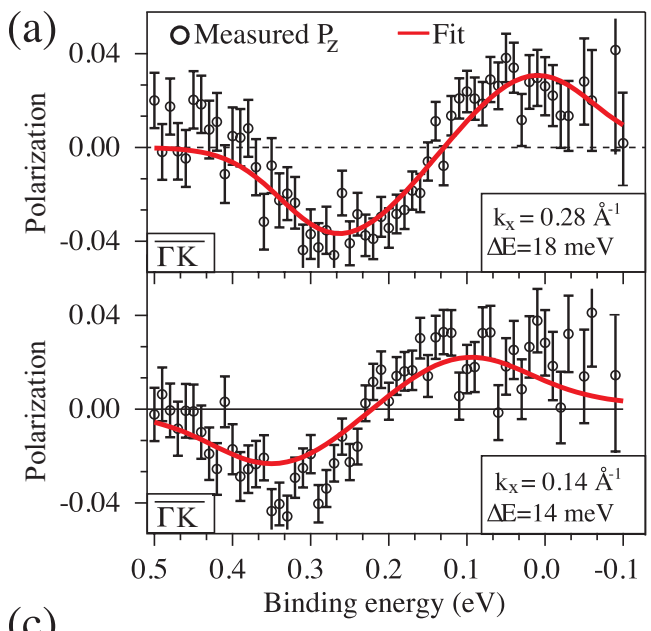

(c)

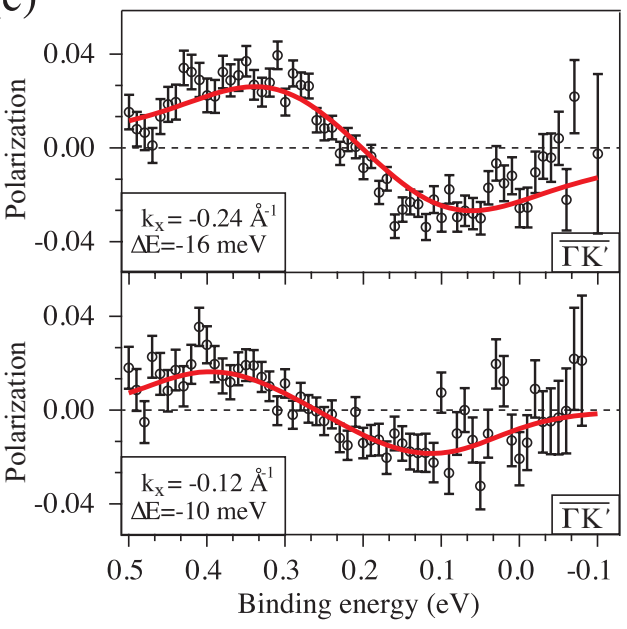

(b)

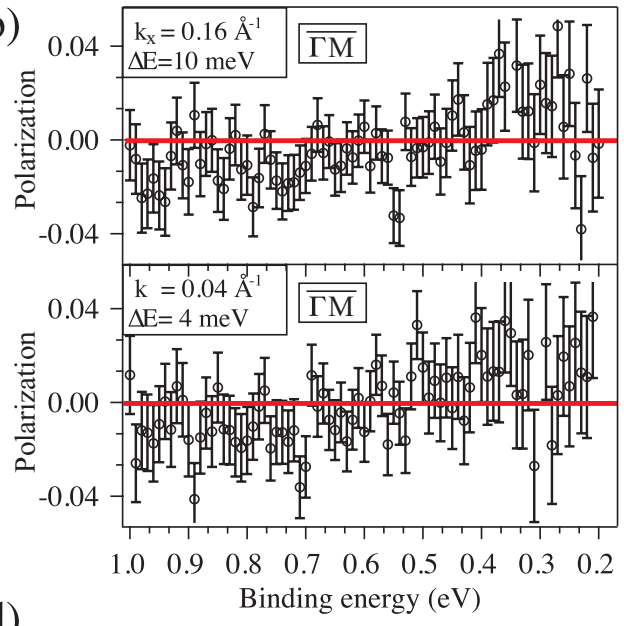

(d)

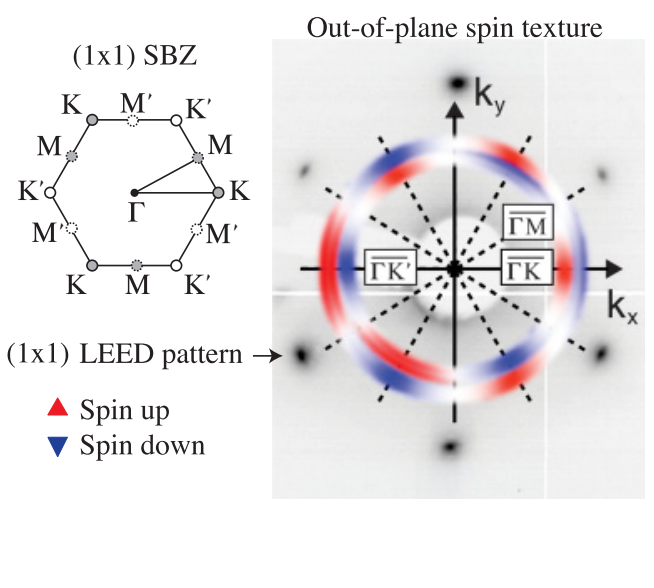

FIG. 4. (Color online) (a)-(c) Measured and fitted $P_{z}$ data for different high symmetry directions of the $(1 \times 1) \mathrm{SBZ}$ of $\mathrm{Pb} / \mathrm{Bi} / \mathrm{Si}(111)$ along: (a) $\overline{\Gamma K}$, (b) $\overline{\Gamma M}$, and (c) $\overline{\Gamma K^{\prime}}$. (d) SBZ of the (111) surface and spin texture of $P_{z}$ overlayed with a LEED pattern of the threefold symmetric $\mathrm{Pb}(111)$ surface.

interface reduces the coupling of the $\mathrm{Pb}$ overlayer to the $\mathrm{Si}$ substrate underneath. The $\mathrm{Pb}$ film becomes more free-standing-like, and it is expected that the Rashba effect of QWS in completely free-standing films vanishes due to the symmetric confinement. ${ }^{23,24}$ A possible system featuring this property is $\mathrm{Pb}$ on an $\mathrm{Ag}$ reconstructed $\mathrm{Si}$ substrate. Figure 3(d) shows the band dispersion of $8 \mathrm{ML} \mathrm{Pb}$ with $m_{\mathrm{Ag}}^{\star}=2.4 m_{e}$. Note that the effective mass in this system is even further reduced compared to $\mathrm{Pb} / \mathrm{Bi} / \mathrm{Si}(111)$. The measured tangential polarizations are displayed in Fig. 3(e) and show no Rashba-type spin splitting within the experimental resolution.

Figure 3(f) compares spin-resolved band dispersions of QWS for all three interfaces, calculated from the experimentally determined $\alpha_{\mathrm{RB}}$ and $m^{\star}$. The difference in effective mass $\left(m_{\mathrm{Pb}}^{\star} / m_{\mathrm{Bi}}^{\star} \approx 3\right)$ together with the reduced value of $\alpha_{\mathrm{RB}}$ cause a reduction of the momentum splitting $k_{0}=m^{\star} \alpha_{\mathrm{RB}} / \hbar^{2}$ by a factor of 8 from $k_{0, \mathrm{~Pb}}=(0.10 \pm 0.002) \AA^{-1}$ to $k_{0, \mathrm{Bi}}=$ $(0.0126 \pm 0.002) \AA^{-1}$.

The $P_{z}$ curve shown in Fig. 2(c) was measured in the high symmetry direction $\overline{\Gamma K}$ of the hexagonal $(1 \times 1)$ SBZ of $\mathrm{Pb} / \mathrm{Bi} / \mathrm{Si}(111)$. This component has to fulfill time-reversal symmetry (TRS), that is, $\mathbf{P}_{z}\left(\mathbf{k}_{\|}\right)=-\mathbf{P}_{z}\left(-\mathbf{k}_{\|}\right)$and since SOI couples the spin to the lattice it also has to reflect the $C_{3 v}$ symmetry of the surface. Figures $4(a)-4$ (c) display $P_{z}$ data taken at different high symmetry directions and polar angles of the $(1 \times 1)$ SBZ. Figure 4 (a) correspond to $\overline{\Gamma K}$ and Fig. 4(c) to $\overline{\Gamma K^{\prime}}$ as sketched in Fig. 4(d). In accordance with TRS we observe a sign change of $P_{z}$. The $P_{z}$ curves shown in Fig. 4(b) have been measured on the QWS in $\mathrm{Pb} / \mathrm{Bi} / \mathrm{Si}(111)$ displayed in Fig. 3(b) (scans 2 and 3) along $\overline{\Gamma M}$. Here no significant $P_{z}$ can be observed within the experimental resolution.

Currently different mechanisms have been discussed to explain an out-of-plane polarization of spin-orbit split states. For topological insulators the inclusion of third order correction in $\mathbf{k}$ to explain the warping of the surface states also induces an out-of-plane component of the spin expectation value. ${ }^{25-27}$ Furthermore, spin frustration at the edge of the Brillouin zone can lead to an abrupt out-of-plane rotation of the Rashba spin. ${ }^{28}$ Because in $\mathrm{Pb} / \mathrm{Bi} / \mathrm{Si}(111)$ the constant energy surfaces of the $6 p_{z}$ derived states show no sign of warping and we are far from the edge of the Brillouin zone $\left(k_{\max } \approx 0.25<\right.$ $0.64 \AA^{-1}$ ), we propose that our observations are related to those for surface alloys [e.g., $\mathrm{Bi} / \mathrm{Ag}(111)$ ] where an out-of-plane spin component is induced by an in-plane potential gradient or an in-plane asymmetric charge distribution..$^{21,29}$ Our previous photoemission measurements show that after deposition of $\mathrm{Pb}$ 
on the $\mathrm{Bi}$ reconstructed $\mathrm{Si}(111)$ the $\mathrm{Bi} 5 d$ core levels shift by $350 \mathrm{meV}$ toward lower binding energy, which indicates a charge transfer between the $\mathrm{Bi}$ atoms and the $\mathrm{Pb}$ overlayer. ${ }^{18}$ Therefore the presence of the $\mathrm{Bi}$ interface influences the standing electron waves in the $\mathrm{Pb}$ layer and the in-plane charge density distribution around a $\mathrm{Pb}$ core will also reflect the $(\sqrt{3} \times \sqrt{3}) R 30^{\circ}$ reconstruction of the interface and its reduced symmetry. The absence of any out-of-plane polarizations in $\mathrm{Pb} / \mathrm{Pb} / \mathrm{Si}(111)$ may result from the destruction of the $\mathrm{Si}(111)-$ $(\sqrt{3} \times \sqrt{3})-\mathrm{Pb}$ periodicity by burial as reported by Howes ${ }^{30}$ and later by Feng. ${ }^{31}$ Apparently the Bi interface maintains its symmetry upon deposition of $\mathrm{Pb}$ at low temperature. A x-ray scattering experiment could clarify this issue.
To conclude, we have shown that the spin splitting and spin texture of $\mathrm{Pb} \mathrm{QWS}$ on $\mathrm{Si}(111)$ can be manipulated by altering the interface. Specifically we observe for $\mathrm{Pb} / \mathrm{Bi} / \mathrm{Si}(111)$ the appearance of an out-of-plane spin component and the reduction of the Rashba parameter. The rotation of the spin polarization out of the surface plane introduces an additional degree of freedom for the spin injection into a Rashba system. The system $\mathrm{Pb} / \mathrm{Ag} / \mathrm{Si}$ (111) features no Rashba-type spin splitting within our experimental resolution.

We thank C. Hess, F. Dubi, M. Kropf, and M. Klöckner for technical support. This work was supported by the Swiss National Foundation.
${ }^{1}$ Y. A. Bychkov and E. I. Rashba, JETP Lett. 39, 78 (1984).

${ }^{2}$ S. A. Wolf, D. D. Awschalom, R. A. Buhrman, J. M. Daughton, S. von Molnár, M. L. Roukes, A. Y. Chtchelkanova, and D. M. Treger, Science 294, 5546 (2001).

${ }^{3}$ J. Nitta, T. Akazaki, H. Takayanagi, and T. Enoki, Phys. Rev. Lett. 78, 1335 (1997).

${ }^{4}$ S. LaShell, B. A. McDougall, and E. Jensen, Phys. Rev. Lett. 77, 3419 (1996).

${ }^{5}$ Yu. M. Koroteev, G. Bihlmayer, J. E. Gayone, E. V. Chulkov, S. Blügel, P. M. Echenique, and Ph. Hofmann, Phys. Rev. Lett. 93, 046403 (2004).

${ }^{6}$ C. R. Ast, D. Pacilé, L. Moreschini, M. C. Falub, M. Papagno, K. Kern, M. Grioni, J. Henk, A. Ernst, S. Ostanin, and P. Bruno, Phys. Rev. B 77, 081407 (2008).

${ }^{7}$ J. H. Dil, J. Phys. Condens. Matter 21, 403001 (2009).

${ }^{8}$ I. Gierz, T. Suzuki, E. Frantzeskakis, S. Pons, S. Ostanin, A. Ernst, J. Henk, M. Grioni, K. Kern, and C. R. Ast, Phys. Rev. Lett. 103, 046803 (2009).

${ }^{9}$ K. Yaji, Y. Ohtsubo, S. Hatta, H. Okuyama, K. Miyamoto, T. Okuda, A. Kimura, H. Namatame, M. Taniguchi, and T. Aruga, Nat. Commun. 1, 17 (2010).

${ }^{10}$ T.-C. Chiang, Surf. Sci. Rep. 39, 181 (2000)

${ }^{11}$ J. H. Dil, F. Meier, J. Lobo-Checa, L. Patthey, G. Bihlmayer, and J. Osterwalder, Phys. Rev. Lett. 101, 266802 (2008).

${ }^{12}$ G. Bihlmayer, Y. M. Koroteev, P. M. Echenique, E. V. Chulkov, and S. Blügel, Surf. Sci. 600, 3888 (2006).

${ }^{13}$ M. Nagano, A. Kodama, T. Shishidou, and T. Oguchi, J. Phys. Condens. Matter 21, 064239 (2009).

${ }^{14}$ K. J. Wan, T. Guo, W. K. Ford, and J. C. Hermanson, Phys. Rev. B 44, 3471 (1991)

${ }^{15}$ D. A. Ricci, T. Miller, and T. C. Chiang, Phys. Rev. Lett. 93, 136801 (2004).

${ }^{16}$ M. Hoesch, T. Greber, V. N. Petrov, M. Muntwiler, M. Hengsberger, W. Auwaerter, and J. Osterwalder, J. Electron Spectrosc. Relat. Phenom. 124, 263 (2002).

${ }^{17}$ J. H. Dil, J. W. Kim, S. Gokhale, M. Tallarida, and K. Horn, Phys. Rev. B 70, 045405 (2004).
${ }^{18}$ B. Slomski, F. Meier, J. Osterwalder, and J. H. Dil, Phys. Rev. B 83, 035409 (2011)

${ }^{19}$ M. H. Upton, T. Miller, and T.-C. Chiang, Phys. Rev. B 71, 033403 (2005).

${ }^{20}$ J. H. Dil, J. W. Kim, Th. Kampen, K. Horn, and A. R. H. F. Ettema, Phys. Rev. B 73, 161308 (2006).

${ }^{21}$ F. Meier, H. Dil, J. Lobo-Checa, L. Patthey, and J. Osterwalder, Phys. Rev. B 77, 165431 (2008).

${ }^{22}$ In Ref. 11 a lower $\alpha_{\mathrm{RB}}=(0.04 \pm 0.005) \mathrm{eV} \AA$ has been determined based on measurements with a single Mott detector. This gave only a lower boundary of the Rashba constant. The exact value found here is based on a full 3D measurement using both Mott detectors.

${ }^{23}$ J. H. Dil, T. U. Kampen, B. Hülsen, T. Seyller, and K. Horn, Phys. Rev. B 75, 161401 (2007).

${ }^{24}$ T. Hirahara, K. Miyamoto, A. Kimura, Y. Niinuma, G. Bihlmayer, E. V. Chulkov, T. Nagao, I. Matsuda, S. Qiao, K. Shimada, H. Namatame, M. Taniguchi, and S. Hasegawa, New J. Phys. 10, 083038 (2008).

${ }^{25}$ L. Fu, Phys. Rev. Lett. 103, 266801 (2009).

${ }^{26}$ S.-Y. Xu, Y. Xia, L. A. Wray, S. Jia, F. Meier, J. H. Dil, J. Osterwalder, B. Slomski, A. Bansil, H. Lin, R. J. Cava, and M. Z. Hasan, Science 332, 560 (2011).

${ }^{27}$ S. Souma, K. Kosaka, T. Sato, M. Komatsu, A. Takayama, T. Takahashi, M. Kriener, K. Segawa, and Y. Ando, Phys. Rev. Lett. 106, 216803 (2011).

${ }^{28}$ K. Sakamoto, T. Oda, A. Kimura, K. Miyamoto, M. Tsujikawa, A. Imai, N. Ueno, H. Namatame, M. Taniguchi, P. E. J. Eriksson, and R. I. G. Uhrberg, Phys. Rev. Lett. 102, 096805 (2009).

${ }^{29}$ C. R. Ast, J. Henk, A. Ernst, L. Moreschini, M. C. Falub, D. Pacilé, P. Bruno, K. Kern, and M. Grioni, Phys. Rev. Lett. 98, 186807 (2007).

${ }^{30}$ P. B. Howes, K. A. Edwards, D. J. Hughes, J. E. Macdonald, T. Hibma, T. Bootsma, and M. A. James, Phys. Rev. B 51, 17740 (1995).

${ }^{31}$ R. Feng, E. H. Conrad, M. C. Tringides, C. Kim, and P. F. Miceli, Appl. Phys. Lett. 51, 3866 (2004). 